\title{
Revaluation of Paperless Partograph in the Management of Labor
}

\author{
Abo Bakr A. Khalil", Sayed A. Mostafa, and Ayman D. Mohamed \\ Department of Obstetrics and Gynecology, Faculty of Medicine, Women Health Hospital, Assuit University
}

\begin{abstract}
Background: The paperless partograph refers to monitoring progress of labor and reaching to an accurate decision for intervention to ensure safe delivery. It needs no graph paper, no extra time to do in comparison to routine partograph. Aim: to reevaluate effect of using paperless partograph on the management and outcome of labor at to prevent prolonged labor and its complications and improving quality of labor in Labor ward at women health hospital Assiut university. Subjects and Methods: The study was conducted from 1st March 2018 to last February 2019 on Women Health Hospital, Assiut University, Egypt, at reception (emergency) unit and it was prospective observational analytical study. Sample of 800 women who met the criteria of; gestational age from 37 to 42 weeks, singleton pregnancy and with cephalic presentation. The data were collected by using the paperless partograph model. Results: Mean age of the participants was 25.6 years and mean of gestational age 39.1 weeks and $87 \%$ were multipara. Mean duration for delivery after Alert ETD was $3.5 \pm 2.1$ hours in primigravida and 3.3 \pm 2.1 hours in multipara. Mean Apgar score of the new-born after 5 minutes was 9.4. Conclusion: Paperless partograph was found to be effective positively on the management and the outcome of labor.
\end{abstract}

Keywords: Management of labor, paperless partograph, labor outcome

\section{Introduction}

Preventing prolonged labor is a cornerstone in reducing maternal and neonatal morbidity and mortality ${ }^{(1)}$. Partograph is a graphic recording of the progress of labor and the condition of the mother and fetus against time. The idea is dating back to Friedman (1957) $)^{(2)}$ who introduced the concept of graphically recording the cervical dilatation during labor. Philpott \& Castle $(1972)^{(3)}$ developed this concept into a tool for labor monitoring by adding the socalled "alert " and "action" lines to the graph. In the original WHO partograph, progress of labor is monitored by recording cervical dilatation, fetal head descent and uterine contractions. Fetal condition is monitored by recording fetal heart rate, condition of membranes and liquor and molding of fetal skull bones. Maternal condition is monitored by recording temperature, pulse rate and blood pressure of the mother, in addition to fluid and drug intake and urine output and analysis for sugar, protein and acetone. Partograph is a very nice comprehensive observational record, and one of the most important advances in modern obstetric care. WHO recommends its universal use as a necessary tool in the 
management of labor? Unfortunately, WHO partograph did not gain wide acceptance neither in the developed nor in low resource countries, because it may need more workload and plotting, more time, more staff and more efforts on training and learning $(4,5)$. Trying to make life easier, WHO provides a modified partograph in which the latent phase is completely omitted and monitoring of labor starts with the start of active phase. However, that modified WHO partograph proved to be a poor document. It is equal or even less equal to the original WHO partograph. Therefore Dr. Debdas(5) has proposed a new, low-skill method, namely, paperless partograph. He claims that this model is a simple tool that needs no graph paper, and no extra time to do. It considers the two basic data on which the original partograph works, namely; the alert line \&the action line ${ }^{(5)}$. This method involves only finding both the alert Expected Time of delivery ETD and action ETD and then adhering to that without any recurrent plotting of data and its interpretation ${ }^{(6)}$. The current study aimed to reevaluate, prospectively, the effect of using the paperless partograph in the management and outcome of labor to prevent prolonged labor and its complications and improving the quality of management of labor inside labor room in women health hospital, Assiut university

\section{Patients and Method}

Study place: The study was carried out at labor word (reception unit) of women health hospital, Assiut University. The study was conducted from 1 march, 2018 to Last February 2019, the reception unit consist of 5 rooms one of them is examination room with a capacity of 3 bed, one other is prenatal room with a capacity of 4 bed, other is postpartum room with a capacity of 8 bed and two are operation rooms, Works in it 14 resident obstetricians

Study design and duration. This study was a prospective observational, analytical study designed to reevaluate the using of paperless partograph in labor ward at women health hospital Assiut university during the period of 12 months from 1 March 2018 to last February 2019.

\section{Study subjects}

The Purposive random sample of women who were in actual labor in active phase of labor or when at cervical dilated $4 \mathrm{~cm}$ or more be included in the study. All women with inclusion criteria were included. Women with exclusion criteria were excluded. Inclusion criteria included women actually in active phase of labor (cervical dilatation is $4 \mathrm{~cm}$ or more) were included if they fulfill the following inclusion criteria: Primi or multi parous women, pregnant 37 weeks up to full term. Single living fetus with cephalic presentation with spontaneous labor. Exclusion criteria included malpresentation, medical complication as hypertension, diabetes, anemia or heart disease, previous obstetrical complication, uterine surgery as caesarean section or myomectomy, multiple pregnancy, intrauterine fetal death, preterm fetus, major fetal anomalies, Placenta prervia or ante-partum hemorrhage of any cause, induced labor, and women with pathological cardio tocograph.

\section{Methods}

Data were obtained by following women during labor. A structured interviewing questionnaire was developed by the researcher and used to collect the socio demographic data, and obstetric history. On admission and after explanation of the role of paperless partograph in labor, all participants were subjected to: 
History

This included personal history (Name, Age, Occupation, Marital status, Special habits, and Address. Menstrual history and first day of last menstrual period, Obstetrical history (Parity, Gravidity and mode of previous delivery or abortion, previous C.S or multiple pregnancy. Complaint(s) and present history. Past history of diabetes, hypertension, cardiac problems, renal problems, bleeding disorder. All women were subjected to general \& Obstetric examination and Blood group testing.

The paperless partograph

This is a simple, inexpensive tool to provide a continuous pictorial overview of labor. It is a technique used for labor management, only after the cervical dilatation reach $4 \mathrm{~cm}$ or more for monitoring the progress of labor until delivery and arriving at the accurate time to intervene for ensuring a safe delivery. It is used by clinicians through calculation of two times, an ALERT ETD (estimated time of delivery) and an ACTION ETD. The ALERT calculation uses Friedman's widely accepted rule that the cervix dilates $1 \mathrm{~cm}$ per hour while a woman is in active labor (Friedman's 1955) ${ }^{(2)}$. Once cervical dilation reaches 4 centimeters and 3 or 4 contractions happen every ten minutes, a woman is active stage of labor. The clinician simply added 6 hours to the time at which the woman became dilated to $4 \mathrm{~cm}$ to find the ALERT ETD. (When cervical dilation is at $10 \mathrm{~cm}$ ). If no labor occurred, the clinician added another 4 hours to the ALERT ETD to get the ACTION ETD. both ETDS should be written in big letters on a woman's case management sheet, questionnaire, the ACTION ETD circled in red (stamped red) and the alert ETD circled in blue (stamped blue). Assessment outcome of maternal and newborn condition as mode of delivery, birth outcome, birth, weight, Apgar score, and any complication may raise for mother or newborn. The primary outcome denotes the average time for delivery among primigravida and multipara after the alert line. While the secondary outcomes denote the number of normal vaginal deliveries, number of cesarean section and their causes related to secondary arrest of descant of head or due to cervical dilation, neonatal outcomes regarding weight and APGAR scores

\section{Statistical Analysis}

The data were tested for normality using the Anderson-Darling test and for homogeneity variances prior to further statistical analysis. Categorical variables were described by number and percent ( $N, \%$, where continuous variables described by mean and standard deviation (Mean, SD). Chi-square and fisher exact tests used to compare between categorical variables where compare between continuous variables by unpaired t-test. A two-tailed p 0.05 was considered statistically significant. All analyses were performed with the IBM SPSS 20.0 software.

\section{Ethical Considerations}

The research proposal was approved from ethical committee in the faculty of medicine. The study followed common ethical principles in clinical research of the administrative design before conducting the study. An authorized permission will be obtained by submission of an official letter from the faculty of Medicine, Assuit university to the responsible authorities of the study setting (women health hospital, Assiut university) to obtain the authorization for data collection. The aim of the study will be explained to every woman before participation, and voluntary participation will be emphasized and an oral consent be obtained, however; they have the right to withdraw from the study at any time. Data 
collection will be anonymous, and confidentiality of the data be secured. there were no risks for study subject during application of the research. The Study subject privacy would be considered during collection of data. written consent be obtained from women or guidance that are willing to participate in the study

\section{Results}

Characteristics of the study participants

The present study enrolled 800 laboring women 696 multi para and 104 primpara, their ages ranged from 18 to 40 years with average $25.6 \pm 5$ years and their average parity was $2 \pm 1.3$. Regarding the gravidity $13 \%$ of participants were primi gravida. The gestational age at onset of labor ranged from 37 to 42 weeks or full term with mean $\pm \mathrm{SD}=39.1 \pm 1.86$. Assessment of the women at onset of labor revealed that their aver age temperature was $37.1 \pm 0.1$, their average heart rate was76.6 \pm 7.5 beats per minute, and their average diastolic blood pressure was $75.1 \pm 9.8 \mathrm{mmHg}$, their average systolic blood pressure was $107.5 \pm 9.9$ $\mathrm{mmHg}$. Uterine contractions had an average of $4.6 \pm 1.2$ contractions per 10 minutes. The maternal urine output ranged from 500 to $800 \mathrm{ml}$ with the mean of $662.5 \pm 84.3$ $\mathrm{ml}$. The time elapsed from the rupture of membranes to the delivery was ranged from 30-210 minutes with average $55 \pm 42.2$ minutes (Table 1).

Average time for delivery among primigravida and multipara after the alert line The average time for delivery among primigravida women after the alert ETD was 3.5 \pm 2.1 hrs. (range 1 to $6 \mathrm{hrs}$ ), while the average time for delivery among the multi parous women after the alert ETD was $3.3 \pm 2.1$ hrs. (range 15 min to $6 \mathrm{hrs}$ ) (Table 2 ).

\begin{tabular}{|l|c|c|}
\hline \multicolumn{3}{|c|}{ Table 1: Characteristics of the study participants } \\
\hline Variable & Range & Mean \pm SD \\
\hline Age (years) & $18-40$ & $25.6 \pm 5$ \\
\hline Parity & $1-7$ & $2 \pm 1.3$ \\
\hline Gestational age (weeks) & $37-42$ or full term & $39.1 \pm 1.86$ \\
\hline Temperature cs & $36.9-37.5$ & $37.1 \pm 0.1$ \\
\hline Pulse (bpm) & $61-90$ & $76.6 \pm 7.5$ \\
\hline Diastolic blood pressure $(\mathrm{mmHg})$ & $60-90$ & $75.1 \pm 9.8$ \\
\hline Systolic blood pressure $(\mathrm{mmHg})$ & $90-120$ & $107.5 \pm 9.9$ \\
\hline Number of uterine contractions / 10 mim. & $3-5$ & $4.6 \pm 1.2$ \\
\hline Urine output (ml) & $500-800$ & $662.5 \pm 84.3$ \\
\hline FHR (bpm) & $110-160$ & $142.6 \pm 12.3$ \\
\hline Time from rupture of membranes to delivery (min) & $30-210$ & $55 \pm 42.2$ \\
\hline
\end{tabular}

\begin{tabular}{|l|c|c|}
\hline \multicolumn{3}{|c|}{$\begin{array}{c}\text { Table 2: The average time for delivery among primigravida } \\
\text { and multipara after the alert line. }\end{array}$} \\
\hline Variable & Range hours, $\mathrm{h}$ & Mean $\pm \mathrm{SD}$ \\
\hline Primi gravida & $1 \mathrm{~h}-6 \mathrm{~h}$ & $3.5 \pm 2.1$ \\
\hline Multi para & 15 minutes-4 houres & $3.3 \pm 2.1$ \\
\hline All women & 15 minutes-6 houres & $3.4 \pm 2.1$ \\
\hline
\end{tabular}

Mode of deliveries

As regarding the mode of delivery all cases 800 cases of whom spontaneously vaginal delivery 622 cases with Percentage $77.75 \%$ and regarding to vaginal delivery with episiotomy 83 cases with Percentage 10. 375\% 


\begin{tabular}{|l|c|c|c|c|}
\hline \multicolumn{5}{|c|}{ Table 3: Modes of deliveries among participants } \\
\hline Variable & primgravida & multipara & total cases & percentage \\
\hline vaginal delivery & 61 & 561 & 622 & $77.75 \%$ \\
\hline vaginal delivery with episitomy & 31 & 52 & 83 & $10.375 \%$ \\
\hline Lower segment c s & 12 & 83 & 95 & $11.875 \%$ \\
\hline total & 104 & 696 & 800 & $100 \%$ \\
\hline
\end{tabular}

\section{Causes of cesarean sections}

About 95/800 cases had lower segment c. section (12 primigravida and 83 multipara). As regarding to lower segment c. section due to secondary arrest of cervical dilatation 64 cases with percentage $8 \%$ of whom 8 cases prim para with percentage $1 \%$ and
56 cases multipara with apercentage $7 \%$, as regarding to lower segment c. section due to Secondary arrest of head descant with full cervical dilatation 31 cases with percentage $3.875 \%$ of whom 4 cases prim para with percentage0.5\% and 27 cases multi para with percentage $3.375 \%$ (Table 4 ).

\begin{tabular}{|l|c|c|c|}
\hline \multicolumn{4}{|c|}{ Table 4: Causes of cesarean section among participants } \\
\hline $\begin{array}{l}\text { causes of lower } \\
\text { segment C. S }\end{array}$ & $\begin{array}{c}\text { Secondary arrest of } \\
\text { cervical dilatation }\end{array}$ & $\begin{array}{c}\text { Secondary arrest } \\
\text { of head descant }\end{array}$ & Total \\
\hline Primpara & 8 cases & 4 cases & 12 cases \\
\hline Multipara & 56 cases & 27 cases & 83 cases \\
\hline Total & 64 cases & cases31 & 95 cases \\
\hline Percentage & $8 \%$ & $3.875 \%$ & $11.875 \%$ \\
\hline
\end{tabular}

\section{Neonatal outcomes}

Regarding the neonatal outcome, the average weight of the new-born was 3631.9 $\pm 344.4 \mathrm{gm}$. The average Apgar score after 1 minute and 5 minutes are $(7.3 \pm 1 \& 9.4$ \pm 0.9 respectively) (Table 5 ).

\begin{tabular}{|l|c|c|}
\hline \multicolumn{3}{|c|}{ Table 5: Neonatal outcomes } \\
\hline Variable & Range & Mean \pm SD \\
\hline Neonatal weight $(\mathrm{gm})$ & $2865-3950$ & $3631.9 \pm 344.4$ \\
\hline Apgar score after 1 minute & $8-10$ & $7.3 \pm 1$ \\
\hline Apgar score after 5 minutes & $8-10$ & $9.4 \pm 0.9$ \\
\hline
\end{tabular}

\section{Discussion}

Base line data were similar to results that done by Mohammed et al $2018^{(7)}$. They enrolled 370 laboring women with average age of $25.1 \pm 5.4$ year (range 19 to 40 years old). Also, these results were slightly similar to the finding conducted in Labor ward at Maternity Hospital affiliated to Ain
Shams University, Egypt which used the paperless partograph for out of 100 laboring women who participated in the study which revealed that their ages ranged from 18 to 35 years with average $25.6 \pm 5$ years ${ }^{(8)}$. The gestational age of participants at onset of labor ranged from 37 to 40 weeks (39.1 \pm 1.86$)$ which is slightly similar to Fatouh \& Ramadan $(2015)^{(8)}$ where 
gestational age ranged from 38-41 weeks (39 \pm 0.9$)$. Regarding the mode of delivery, the present study revealed that 800 cases of whom normal delivery 622 cases with percentage $77.75 \%$ and episiotomy normal delivery 83 cases with percentage $10.375 \%$ and lower segment $C$-section 95 cases with percentage $11.875 \%$. These findings were nearly corresponding with another study ${ }^{(8)}$ that used the paperless partograph for the management of labor revealed that the most of the women had normal vaginal delivery ( $88 \%)$, whereas only $12 \%$ of them had caesarean section. As regards to the average time for delivery the study revealed that among the primigravida women after the alert ETD was $3.5 \pm 2.1$ hours (ranged from 1 to 6 hours), while the average time for delivery among the multiparous women after the alert ETD was $3.3 \pm 2.1$ hours ranged from 15 minutes to 6 hours. These result was similar to the result of (Mohammed et al., 2018)(7) which was 4.3 hours in that study, the mean duration for delivery after alert line ETD was $4.7 \pm 1.9$ hours in primigravida and $3.7 \pm 1.8$ hours in multipara, however, these differences were not statistically significant, and was similar to the WHO recommendation for partograph with a four-hour action line denoting the timing of intervention for prolonged labor(9). Also these findings were nearly corresponding with other study which used the paperless partograph for the management of labor(6); which was shown to be effective in preventing prolonged labor. Most of cases in the current study not reached to action line and those who reached to it were given the appropriate care in suitable time, so none of them were complicated by prolonged labor. Regarding $\mathrm{C}$-section due to secondary arrest of cervical dilatation, 64 cases $8 \%$ of whom 8 cases prim para $1 \%$ and 56 cases multipara with $7 \%$. Regarding to lower segment c. section due to Secondary arrest of head descant, 31cases $3.875 \%$ of whom 4 cases prim para with a ratio $0.5 \%$ and 27 cases multi para $3.375 \%$ woman whose labor was not progressing were transferred and managed in third floor emergency operative unit in same women health hospital by senior obstetrician. Regarding to the neonatal outcome, all the new-born babies weights were within normal. The average Apgar score after 1 minute and 5 minutes are $7.3 \pm 1 \& 9.4 \pm 0.9$ respectively; this result was interpreted as there was no newborn need to admit to Neonate Intensive Care Unit ( $\mathrm{NICU}$ ) nor need ventilation. This result revealed the positive effect of paperless partograph on neonatal outcome. These results were nearly corresponding to the study findings conducted by (Fatouh \& Ramadan, 2015) the average weight of the new-born ranged from $3.6 \pm 0.4 \mathrm{~kg}$ and $51 \%$ of them were females and $49 \%$ males ${ }^{(8)}$. The use of paperless partograph in labor management very simple and not needs graph paper or extra time to do it, but the WHO partograph is very complicated and require more training before using it. This finding was supported by Debdas $2008^{(5)}$ whom argues that the partograph of WHO fails to meet the organization's own requirements for appropriate technology and has not been adapted to local needs, is not acceptable to those who use it and too complicated for many skilled birth attendants. One study found that less than $10 \%$ of providers (nurses, midwives, and community health care workers) routinely use the partograph in peripheral centers in Nigeria (Oladapo, $O$ et al., 2006) ${ }^{(10)}$. The use of paperless partograph in labor management was very simple and did not need graph paper or extra time to do it, but the WHO partograph was very complicated and requires more training before using it. Also it was reported that despite the proven effectiveness of traditional partograph, some members pointed out they 
were not frequently used at all health care facility level(5). Additionally, The Paperless partograph attempted to provide a lowcost, intuitive solution to many of the problems facing effective partograph use in the developing world. So, the findings of present study supported the research hypothesis which the view that use of the paperless partograph would be easy and effective design for the management and outcome of labor.

\section{Conclusions}

We concluded that; the paperless partograph was found convenient and effective in the management of labor and shown to be effective in preventing prolonged labor and improving the maternal and neonatal outcome and should be implemented as essential part of care in all health facilities.

\section{References}

1. Lavender, T., Hart, A. \& Smyth, R. (2008). Effect of partograph use on outcomes for women in spontaneous labor at term. Cochrane Database of Systematic Reviews. 2008 (4): https://doi.org/10.1002/14651858..pub2 CD005461

2. Friedman curve 1957. https://www.bellybelly.com.au/birth/friedmans-curvein-labour/

3. Philpott RH, and Castle WM. (1972) Cervicographs in the management of labour in primigravidae. BJOG: Int J Obs \& Gyn, 79, 599-602.
4. World Health Organization (1994). World Health Organization partograph in management of labor. Lancet; 1994. 343:1399-404.

5. Debdas A. (2008). Paperless partograph. 41st Annual Scientific Sessions: Sri Lanka College of Obstetrics and Gynecologists. SLJOG Vol. 30. 2008; 1:124

6. Agarwal, K., Agarwal, L., Agrawal, V., Agarwal, A. \& Sharma, M. (2013). Evaluation of paperless partograph as a bedside tool in the management of labor. J Family Med Prim Care. 2013 JanMar; 2(1): 47-49.

7. Mohammed AA, Fouly HA, Abbas AM, et al. (2018): Effect of Using the Paperless Partograph Versus the original Partograph on Labor Outcomes in Women's Health Hospital. Assiut Scientific Nursing Journal, Vol, (6) No, (13) April 2018

8. Fattouh E, and Ramadan S, (2015): Effect of using Paperless Partogram on the Management and Outcome of Labor and the Nurses' Opinion. J Edu Practice.;6(8):17-23.

9. Banerjee M. and Sharma D. (2008). Maternal and Child Health Community. Solution Exchange for the Maternal and Child Health Community. Discussion Summary. Promoting Active Management of Third Stage Labor (AMTSL). Int J Gyn and Obs. 2007.7 (1). 1: 4-

10. Oladapo $O$, Daniel $O$, Olatunji $A$. Knowledge and use of the partograph among health care personnel at the peripheral maternity centers in Nigeria. $J$ Obstet Gynaecol 2006; 26(6):538-541 